Peng-Peng Li, Jin-Xia Ma, Qing-Lin Li, Ji-Xing Zhao and Li Zhao*

\title{
Crystal structure of 2-((E)-((4-((E)-1- (hydroxyimino)ethyl)phenyl)iminio)methyl)- 5-methoxyphenolate, $\mathrm{C}_{16} \mathrm{H}_{16} \mathrm{~N}_{2} \mathrm{O}_{3}$
}

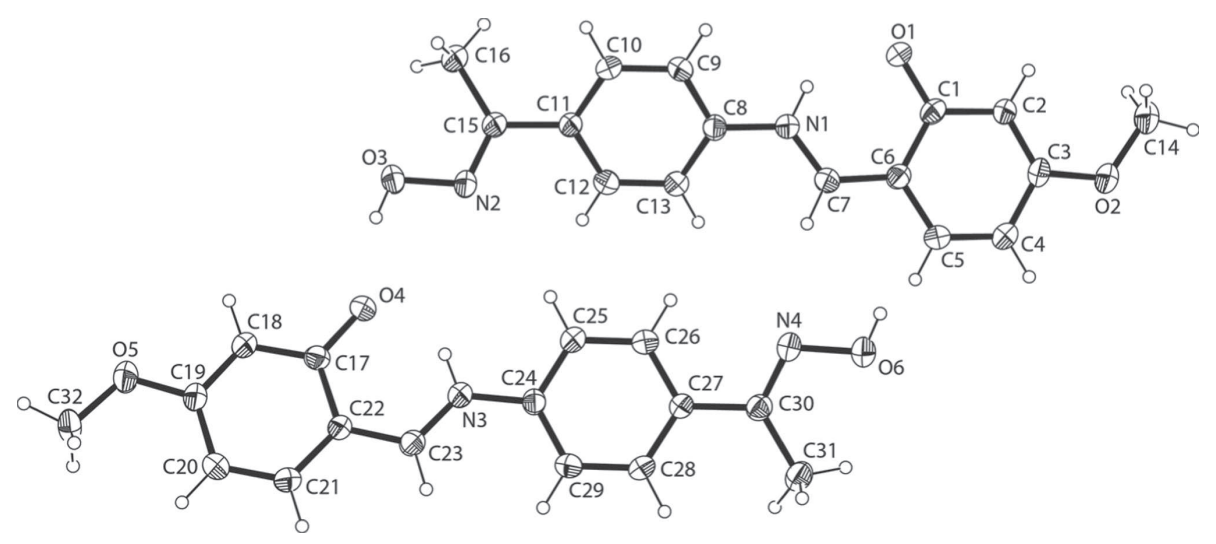

https://doi.org/10.1515/ncrs-2017-0384

Received February 13, 2018; accepted May 14, 2018; available online June 13, 2018

\section{Abstract}

$\mathrm{C}_{16} \mathrm{H}_{16} \mathrm{~N}_{2} \mathrm{O}_{3}, \quad$ triclinic, $\quad P \overline{1} \quad$ (no. 2), $a=8.7904(7) \AA$, $b=12.0808(11) \AA, \quad c=14.3999(11) \AA, \quad \alpha=68.001(8)^{\circ}$, $\beta=81.114(6)^{\circ}, \quad \gamma=83.933(7)^{\circ}, \quad Z=4, \quad V=1398.9(2) \AA^{3}$, $R_{\mathrm{gt}}(F)=0.0658, w R_{\text {ref }}\left(F^{2}\right)=0.1827, T=290.83(10) \mathrm{K}$.

\section{CCDC no.: 1843123}

The asymmetric unit of the title crystal structure is shown in the figure. Tables 1 and 2 contain details on crystal structure and measurement conditions and a list of the atoms including atomic coordinates and displacement parameters.

\section{Source of material}

All solvents and other reagents were of analytical grade and the title compound was prepared by modification of the reported method $[1,2]$. To an ethanol solution $(5 \mathrm{~mL})$ of 2-hydroxy-4-methoxy benzaldehyde ( $456.5 \mathrm{mg}, 3 \mathrm{mmol}$ )

*Corresponding author: Li Zhao, Lanzhou Jiaotong University, School of Chemical and Biological Engineering, Lanzhou 730070, P.R. China, e-mail: zhaoli_72@163.com

Peng-Peng Li, Jin-Xia Ma, Qing-Lin Li and Ji-Xing Zhao: Lanzhou Jiaotong University, School of Chemical and Biological Engineering, Lanzhou 730070, P.R. China

Table 1: Data collection and handling.

Crystal:

Size:

Wavelength:

$\mu$ :

Diffractometer, scan mode:

$\theta_{\max }$, completeness:

$N(h k l)_{\text {measured }}, N(h k l)_{\text {unique }}, R_{\text {int }}$ :

Criterion for $I_{\text {obs }}, N(h k l)_{\mathrm{gt}}$ :

$N(\text { param })_{\text {refined }}$ :

Programs:

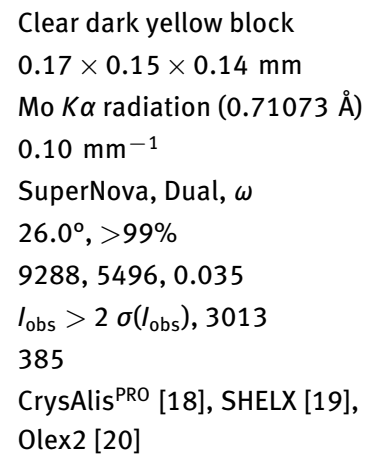

was added an ethanol solution (5 mL) of 1-(4-aminophenyl)ethanone oxime ( $450.6 \mathrm{mg}, 1 \mathrm{mmol}$ ). The solution had been stirred at $328 \mathrm{~K}$ for $7 \mathrm{~h}$, then the mixture was filtered at room temperature. The product was dried under vacuum and a yellow solid of title compound was obtained (yield 81.3\%, m.p. 392-397 K). The crystals were obtained by slow evaporation from a mixture of chloroform/ethanol (1:1) solution in a quiet environment at room temperature. Elemantal analysis: Anal. calcd. for $\mathrm{C}_{16} \mathrm{H}_{16} \mathrm{~N}_{2} \mathrm{O}_{3}$ : C, 67.59\%; H, 5.67\%; N, 9.85\%; Found: C, $67.61 \%$; H, 5.62\%; N, 9.81\%.

\section{Experimental details}

Hydrogen atoms were placed in their geometrically idealized positions and constrained to ride on their parent atoms. 
Table 2: Fractional atomic coordinates and isotropic or equivalent isotropic displacement parameters $\left(\AA^{2}\right)$.

\begin{tabular}{|c|c|c|c|c|}
\hline Atom & $x$ & $y$ & $z$ & $U_{\text {iso }} * / U_{\text {eq }}$ \\
\hline 01 & $0.6910(2)$ & $0.46356(17)$ & $-0.12420(14)$ & $0.0519(6)$ \\
\hline 02 & $0.4414(2)$ & $0.84437(19)$ & $-0.28942(15)$ & $0.0603(6)$ \\
\hline 03 & $1.2073(3)$ & $0.0758(2)$ & $0.51307(15)$ & $0.0667(7)$ \\
\hline H3 & 1.1986 & 0.0978 & 0.5611 & $0.100^{\star}$ \\
\hline N1 & $0.7900(3)$ & $0.4301(2)$ & $0.04568(16)$ & $0.0457(6)$ \\
\hline H1 & 0.7783 & 0.4023 & 0.0004 & $0.055^{\star}$ \\
\hline N2 & $1.1228(3)$ & $0.1565(2)$ & $0.43782(17)$ & $0.0529(7)$ \\
\hline $\mathrm{C} 1$ & $0.6387(3)$ & $0.5701(3)$ & $-0.1284(2)$ & $0.0434(7)$ \\
\hline $\mathrm{C} 2$ & $0.5637(3)$ & $0.6489(2)$ & $-0.2105(2)$ & $0.0447(7)$ \\
\hline $\mathrm{H} 2$ & 0.5504 & 0.6246 & -0.2627 & $0.054^{\star}$ \\
\hline C3 & $0.5107(3)$ & $0.7607(3)$ & $-0.2132(2)$ & $0.0450(7)$ \\
\hline $\mathrm{C} 4$ & $0.5247(3)$ & $0.7990(3)$ & $-0.1336(2)$ & $0.0530(8)$ \\
\hline H4 & 0.4870 & 0.8748 & -0.1362 & $0.064^{\star}$ \\
\hline C5 & $0.5930(3)$ & $0.7245(3)$ & $-0.0539(2)$ & $0.0518(8)$ \\
\hline H5 & 0.6001 & 0.7495 & -0.0010 & $0.062^{\star}$ \\
\hline $\mathrm{C} 6$ & $0.6543(3)$ & $0.6096(2)$ & $-0.0482(2)$ & $0.0426(7)$ \\
\hline $\mathrm{C} 7$ & $0.7293(3)$ & $0.5368(3)$ & $0.0349(2)$ & $0.0457(7)$ \\
\hline H7 & 0.7361 & 0.5663 & 0.0851 & $0.055^{\star}$ \\
\hline C8 & $0.8733(3)$ & $0.3560(3)$ & $0.1249(2)$ & $0.0417(7)$ \\
\hline C9 & $0.8887(3)$ & $0.2343(3)$ & $0.1446(2)$ & $0.0475(7)$ \\
\hline H9 & 0.8437 & 0.2031 & 0.1059 & $0.057^{\star}$ \\
\hline C10 & $0.9701(3)$ & $0.1587(3)$ & $0.2210(2)$ & $0.0486(8)$ \\
\hline H10 & 0.9797 & 0.0773 & 0.2325 & $0.058^{\star}$ \\
\hline C11 & $1.0375(3)$ & $0.2016(2)$ & $0.28081(19)$ & $0.0400(7)$ \\
\hline C12 & $1.0234(3)$ & $0.3246(3)$ & $0.2589(2)$ & $0.0470(7)$ \\
\hline H12 & 1.0696 & 0.3562 & 0.2968 & $0.056^{\star}$ \\
\hline C13 & $0.9431(3)$ & $0.4003(3)$ & $0.1830(2)$ & $0.0477(8)$ \\
\hline H13 & 0.9355 & 0.4819 & 0.1703 & $0.057^{\star}$ \\
\hline C14 & $0.4177(4)$ & $0.8153(3)$ & $-0.3731(3)$ & $0.0770(11)$ \\
\hline $\mathrm{H} 14 \mathrm{~A}$ & 0.3644 & 0.8815 & -0.4194 & $0.115^{\star}$ \\
\hline H14B & 0.3572 & 0.7462 & -0.3496 & $0.115^{\star}$ \\
\hline $\mathrm{H} 14 \mathrm{C}$ & 0.5156 & 0.7987 & -0.4066 & $0.115^{\star}$ \\
\hline C15 & $1.1218(3)$ & $0.1217(3)$ & $0.3641(2)$ & $0.0446(7)$ \\
\hline C16 & $1.1989(4)$ & $0.0053(3)$ & $0.3617(2)$ & $0.0589(9)$ \\
\hline H16A & 1.2974 & -0.0061 & 0.3859 & $0.088^{*}$ \\
\hline H16B & 1.2130 & 0.0072 & 0.2937 & $0.088^{\star}$ \\
\hline H16C & 1.1355 & -0.0593 & 0.4040 & $0.088^{\star}$ \\
\hline 04 & $1.1123(2)$ & $0.16546(18)$ & $0.65708(14)$ & $0.0587(6)$ \\
\hline 05 & $1.2851(2)$ & $-0.0209(2)$ & $0.97293(16)$ & $0.0668(7)$ \\
\hline 06 & $0.4308(3)$ & $0.68016(19)$ & $0.19696(16)$ & $0.0620(6)$ \\
\hline H6 & 0.4029 & 0.6425 & 0.1661 & $0.093^{*}$ \\
\hline N3 & $0.9266(3)$ & $0.3468(2)$ & $0.62452(17)$ & $0.0444(6)$ \\
\hline H3A & 0.9827 & 0.2939 & 0.6062 & $0.053^{*}$ \\
\hline N4 & $0.5314(3)$ & $0.6069(2)$ & $0.26477(17)$ & $0.0507(7)$ \\
\hline C17 & $1.1143(3)$ & $0.1635(2)$ & $0.7479(2)$ & $0.0416(7)$ \\
\hline C18 & $1.1988(3)$ & $0.0748(3)$ & $0.8172(2)$ & $0.0479(8)$ \\
\hline H18 & 1.2568 & 0.0166 & 0.7974 & $0.057^{\star}$ \\
\hline C19 & $1.1983(3)$ & $0.0713(3)$ & $0.9132(2)$ & $0.0460(7)$ \\
\hline C20 & $1.1126(3)$ & $0.1575(3)$ & $0.9463(2)$ & $0.0518(8)$ \\
\hline $\mathrm{H} 2 \mathrm{O}$ & 1.1127 & 0.1551 & 1.0116 & $0.062^{\star}$ \\
\hline C21 & $1.0290(3)$ & $0.2450(3)$ & $0.8799(2)$ & $0.0489(8)$ \\
\hline $\mathrm{H} 21$ & 0.9717 & 0.3022 & 0.9014 & $0.059^{\star}$ \\
\hline $\mathrm{C} 22$ & $1.0260(3)$ & $0.2522(2)$ & $0.7804(2)$ & $0.0399(7)$ \\
\hline C23 & $0.9345(3)$ & $0.3415(3)$ & $0.7155(2)$ & $0.0442(7)$ \\
\hline $\mathrm{H} 23$ & 0.8784 & 0.3982 & 0.7382 & $0.053^{\star}$ \\
\hline
\end{tabular}

Table 2 (continued)

\begin{tabular}{lrrrr}
\hline Atom & $\boldsymbol{x}$ & $\boldsymbol{y}$ & $\boldsymbol{z}$ & $\boldsymbol{U}_{\text {iso }}{ }^{*} \boldsymbol{U}_{\text {eq }}$ \\
\hline C24 & $0.8375(3)$ & $0.4281(3)$ & $0.5522(2)$ & $0.0399(7)$ \\
C25 & $0.8260(3)$ & $0.4011(3)$ & $0.4678(2)$ & $0.0493(8)$ \\
H25 & 0.8784 & 0.3334 & 0.4603 & $0.059^{*}$ \\
C26 & $0.7374(3)$ & $0.4744(3)$ & $0.3955(2)$ & $0.0491(8)$ \\
H26 & 0.7298 & 0.4548 & 0.3398 & $0.059^{*}$ \\
C28 & $0.6762(3)$ & $0.6056(3)$ & $0.4867(2)$ & $0.0467(7)$ \\
H28 & 0.6284 & 0.6754 & 0.4928 & $0.056^{*}$ \\
C29 & $0.7640(3)$ & $0.5313(3)$ & $0.5604(2)$ & $0.0481(7)$ \\
H29 & 0.7735 & 0.5512 & 0.6156 & $0.058^{*}$ \\
C27 & $0.6584(3)$ & $0.5775(2)$ & $0.4038(2)$ & $0.0406(7)$ \\
C30 & $0.5571(3)$ & $0.6521(3)$ & $0.3282(2)$ & $0.0432(7)$ \\
C31 & $0.4851(3)$ & $0.7692(3)$ & $0.3310(2)$ & $0.0574(9)$ \\
H31A & 0.4615 & 0.8196 & 0.2648 & $0.086^{*}$ \\
H31B & 0.5557 & 0.8076 & 0.3524 & $0.086^{*}$ \\
H31C & 0.3920 & 0.7555 & 0.3775 & $0.086^{*}$ \\
C32 & $1.2793(4)$ & $-0.0378(3)$ & $1.0766(2)$ & $0.0721(11)$ \\
H32A & 1.3206 & 0.0296 & 1.0822 & $0.108^{*}$ \\
H32B & 1.3392 & -0.1092 & 1.1098 & $0.108^{*}$ \\
H32C & 1.1743 & -0.0453 & 1.1078 & $0.108^{*}$ \\
\hline
\end{tabular}

\section{Comment}

The structures of many compounds with oxime-type ligands have been reported, often with di, tri- or polynuclear structures [3-5]. Oximes are an important class of ligands with interesting properties due to the presence of several coordination sites [6-8]. Researchers have focused their interests on complexes, for their extensive applications [9-11]. For example: it has been used to nonlinear optical materials [12-14] and supramolecular architectures [15-17] etc.

The single crystal structure of the title compound was determined by X-ray crystallography. The asymmetric unit of the title structure contains two crystallographically independent but chemically identical molecules ( $c f$. the figure). And all bond lengths are in normal ranges. In the molecules, there are strong intramolecular $\mathrm{N}-\mathrm{H} \cdots \mathrm{O}$ hydrogen bonds $(\mathrm{O} 1-\mathrm{H} 1 \cdots \mathrm{N} 1: \quad \mathrm{d}(\mathrm{N} 1 \cdots \mathrm{O} 1)=2.598 \AA, \quad \mathrm{d}(\mathrm{N} 1-\mathrm{H} 1)=0.86 \AA$

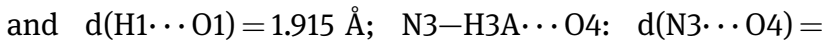
$2.529 \AA, \mathrm{d}(\mathrm{N} 3-\mathrm{H} 3 \mathrm{~A})=0.86 \AA$ and $\mathrm{d}(\mathrm{H} 3 \mathrm{~A} \cdots \mathrm{O} 4)=1.810 \AA$ ). In addition, there are strong intermolecular $\mathrm{O} 3-\mathrm{H} 3 \cdots \mathrm{O} 4$, O6-H6 - O O1 hydrogen bonds formed a chain structure.

Acknowledgements: This work was supported by the Science Foundation of Gansu Education Department of China (2017A-023).

\section{References}

1. Li, P. P.; Zhao, L.; Zhao, J. X.; Zhu, Z. B.: Crystal structure of (E)-1-(4-(( $(E)$-4-(diethylamino)-2-hydroxybenzylidene)amino) phenyl)ethan-1-one oxime, $\mathrm{C}_{19} \mathrm{H}_{23} \mathrm{~N}_{3} \mathrm{O}_{2}$. Z. Kristallogr. NCS 233 (2018) 13-15. 
2. Zhao, L.; Wang, F.; An, Q. Q.; Zhao, J. X.: Crystal structure of (E)1-(4-(( $(E)-3,5$-dichloro-2-hydroxy-benzylidene)amino)phenyl) ethan-1-one $O$-ethyl oxime, $\mathrm{C}_{17} \mathrm{H}_{16} \mathrm{Cl}_{2} \mathrm{~N}_{2} \mathrm{O}_{2}$. Z. Kristallogr. NCS 231 (2016) 1045-1046.

3. Zhao, L.; Zhao, J. X.; An, Q. Q.; Wang, F.: Crystal structure of (E)1-(4-(((E)-3,5-dibromo-2-hydroxybenzylidene)amino)phenyl) ethan-1-one $O$-methyl oxime $\mathrm{C}_{17} \mathrm{H}_{16} \mathrm{Br}_{2} \mathrm{~N}_{2} \mathrm{O}_{2}$. Z. Kristallogr. NCS 231 (2016) 1053-1054.

4. Zhao, J. X.; Zhao, L.; Li, P. P.; Wang, F.; An, Q. Q.: Crystal structure of $(E)-1-(4-(((E)-5$-bromo-2-hydroxybenzylidene) amino)phenyl)ethan-1-one $O$-methyl oxime, $\mathrm{C}_{16} \mathrm{H}_{15} \mathrm{BrN}_{2} \mathrm{O}_{2}$. Z. Kristallogr. NCS 232 (2017) 731-732.

5. Li, P. P.; Zhao, L.; Zhao, J. X.; Zhu, Z. B.; Wang, F.; An, Q. Q.: Synthesis and crystal structure of bis $\{1-(((4-(1-$ (hydroxyimino)ethyl)phenyl)imino)methyl)naphthalene-2olato- $\mathrm{K}^{2} \mathrm{O}, \mathrm{N}$ \} copper(II), $\mathrm{C}_{38} \mathrm{H}_{30} \mathrm{CuN}_{4} \mathrm{O}_{4}$. Z. Kristallogr. NCS 232 (2017) 889-890.

6. Dong, W. K.; Duan, J. G.; Guan, Y. H.; Shi, J. Y.; Zhao, C. Y.: Synthesis, crystal structure and spectroscopic behaviors of $\mathrm{Co}(\mathrm{II})$ and $\mathrm{Cu}$ (II) complexes with Salen-type bisoxime ligands. Inorg. Chim. Acta 362 (2009) 1129-1134.

7. Zhao, J. X.; Zhao, L.; Liu, G. H.; Li, P. P.; Zhu, Z. B.: Crystal structure of 1-\{4-[(5-chloro-2-hydroxy-benzylidene)amino]phenyl\} ethanone $O$-ethyloxime, $\mathrm{C}_{17} \mathrm{H}_{17} \mathrm{ClN}_{2} \mathrm{O}_{2}$. Z. Kristallogr. NCS 232 (2017) 999-1001.

8. Li, P. P.; Zhao, L.; Liu, G. H.; Zhao, J. X.; Zhu, Z. B.: Synthesis and crystal structure of 1-\{4-[(2-hydroxy-benzylidene)amino] phenyl\}ethanone oxime, $\mathrm{C}_{15} \mathrm{H}_{14} \mathrm{~N}_{2} \mathrm{O}_{2}$. Z. Kristallogr. NCS 232 (2017) 1005-1006.

9. Dong, W. K.; Gong, S. S.; Tong, J. F.; Sun, Y. X.; Wu, J. C.: Syntheses and structure of two copper(II) complexes with salicyl mono-oxime ligands. Chin. J. Inorg. Chem. 26 (2010) 1868-1874.

10. Zhao, L.; An, Q. Q.; Zhao, J. X.; Li, P. P.; Wang, F.: Crystal structure of bis(2-((E)-((4-((E)-1-(ethoxyimino)ethyl) phenyl)imino)methyl)-5-methoxy- phenolato- $\mathrm{K}^{2} \mathrm{O}$, N) copper(II), $\mathrm{C}_{36} \mathrm{H}_{38} \mathrm{CuN}_{4} \mathrm{O}_{6}$. Z. Kristallogr. NCS 232 (2016) 595-597.
11. Dong, W. K.; Zhang, X. Y.; Sun, Y. X.; Dong, X. Y.; Li, G.; Wang, J.: A 2D supramolecular copper(II) complex with an asymmetric Salamo-type ligand: Synthesis, crystal structure, and fluorescent property. Synth. React. Inorg. Met.-Org. Nano-Met. Chem. 45 (2015) 956-962.

12. Sun, Y. X.; Zhao, Y. Y.; Li, C. Y.; Yu, B.; Guo, J. Q.; Li, J.: Supramolecular cobalt(II) and copper(II) complexes with Schiff base ligand: Syntheses, characterizations and crystal structures. Chin. J. Inorg. Chem. 32 (2016) 913-920.

13. Dong, W. K.; Li, X. L.; Wang, L.; Zhang, Y.; Ding, Y. J.: A new application of Salamo-type bisoximes: as a relay-sensor for $\mathrm{Zn}^{2+} / \mathrm{Cu}^{2+}$ and its novel complexes for successive sensing of $\mathrm{H}^{+} / \mathrm{OH}^{-}$. Sens. Actuators B 229 (2016) 370-378.

14. Sun, Y. X.; Li, C. Y.; Yang, C. J.; Zhao, Y. Y.; Guo, J. Q.; Yu, B.: Two $\mathrm{Cu}(I I)$ complexes with Schiff base ligands: Synthesis, crystal structure, spectroscopic properties, and substituent effect. Chin. J. Inorg. Chem. 32 (2016) 327-335.

15. Zhao, L.; Dong, X. T.; Sun, Y. X.; Cheng, Q.; Dong, X. Y.; Wang, L.: Synthesis, crystal structure and thermal property of a 1D chain-like copper(II) complex. Chin. J. Inorg. Chem. 28 (2012) 2413-2418.

16. Sun, Y. X.; Lu, R. E.; Li, X. R.; Zhao, Y. Y.; Li, C. Y.: A Schiff base ligand containing oxime group and its $\mathrm{Cu}(\mathrm{II})$ complex: syntheses and supramolecular structures. Chin. J. Inorg. Chem. 31 (2015) 1055-1062.

17. Zhao, L.; Dong, X. T.; Cheng, Q.; Zhao, J. X.; Wang, L.: Synthesis, crystal structure and spectral properties of a $2 \mathrm{D}$ supramolecular copper(II) complex with 1-(4-\{[(E)-3-ethoxyl2-hydroxybenzylidene]amino\}phenyl)ethanone oxime. Synth. React. Inorg. Met.-Org. Nano-Met. Chem. 43 (2013) 1241-1246.

18. CrysAlis ${ }^{\mathrm{PRO}}$ Software System, Version 1.171.37.35, Agilent Technologies UK Ltd, Oxford, UK (2011).

19. Sheldrick, G. M.: A short history of SHELX. Acta Crystallogr. A64 (2008) 112-122.

20. Dolomanov, O. V.; Bourhis, L. J.; Gildea, R. J.; Howard, J. A. K.; Puschmann, H.: OLEX2: A complete structure solution, refinement and analysis program. J. Appl. Crystallogr. 42 (2009) 339-341. 